\title{
Efficacy of conservative treatment of perianal abscesses in children and predictors for therapeutic failure
}

Lars Boenicke, MD, $\mathrm{PhD}^{1, *}$, Johannes Doerner, $\mathrm{MD}^{1, *}$, Stefan Wirth, PhD ${ }^{2}$, Hubert Zirngibl, PhD ${ }^{1}$, Mike Ralf Langenbach, PhD ${ }^{1,3}$

${ }^{1}$ Department of General and Visceral Surgery, Helios University Hospital Wuppertal, University Witten-Herdecke, Wuppertal, Germany; ${ }^{2}$ Department of Pediatrics, Helios University Hospital Wuppertal, University Witten-Herdecke, Wuppertal, Germany; ${ }^{3}$ Department of General and Visceral Surgery and Coloproctology, Helios Clinic Oberhausen, Oberhausen, Germany

Background: The optimal management of perianal abscess in children is controversial.

Purpose: To evaluate the efficiency of conservative treatment of perianal abscess in children and identify parameters that predict therapy failure.

Methods: All cases of children younger than 14 years of age with perianal abscesses between 2001-2016 were evaluated.

Results: Of the 113 enrolled patients, 64 underwent subsequent surgery for advanced disease (primary surgery group). Conservative treatment was initiated in 49 patients (primary conservative group) but was stopped because of inefficiency in 25 patients, who were referred for surgery after a median 7.03 days (range, 2 to 16 days). The other 24 patients (48\%) initially achieved complete remission after conservative treatment, but 10 were readmitted after a median 34 months (range, 3 to 145 months) with recurrent disease. There were no significant differences in permanent success after conservative treatment between infants (10 of 29,34\%) and older children (4 of 20 [20\%], $P=0.122$ ). Overall, conservative treatment alone was effective in only 14 of 113 patients. Recurrence after surgery occurred in 16 patients (25\%) in the primary surgery group and 11 patients $(22 \%)$ in the primary conservative group $(P=0.75)$. Univariate analysis of predictors for conservative treatment failure revealed inflammatory values (C-reactive protein and white blood count, $P=0.017)$ and abscess size $(P=0.001)$ as sig. nificant parameters, whereas multivariate analysis demonstrated that only abscess size (odds ratio, $3.37 ; P=0.023$ ) was significant. Conclusion: Conservative treatment of perianal abscess is permanently efficient in only a minority of children but is not associated with a higher recurrence rate after subsequent surgery. Abscess size is a predictor for therapy failure.

Keywords: Anal fistula, Conservative treatment, Child, Perianal abscess, Recurrence

\section{Key message}

Question: How effective is conservative treatment of perianal abscess in children?

Finding: In cases with early stage disease, short-term remission can be achieved in some patients with a notable risk of recurrence. Abscess size is a predictor for therapy failure. At last, most patients require surgery, but they do not have higher risk for recurrence after ineffective conservative treatment.

Meaning: Conservative treatment should be considered only for small perianal abscess.

\section{Introduction}

The recommendations for management of perianal abscess and fistula in ano in children are inconsistent and differ from those in adults. ${ }^{1-3)}$ In adults, about $90 \%$ of all cases with perianal abscess and fistula in ano are assessed to have cryptoglandular etiology. ${ }^{4}$ Anal fistula and perianal abscess may occur alternately at the same location and are therefore considered to be 2 clinical presentations of a cryptoglandular infection. ${ }^{4,5)}$

In contrast to the adult population, pediatric disease affects almost exclusively boys, is infrequently associated with sepsis and may also resolve spontaneously. ${ }^{6-8)}$ Various approaches have been suggested for their management. The lack of consensus in the treatment of perianal abscess and fistula makes the choice of treatment difficult.9)

While some authors favor primarily surgery, ${ }^{10-12)}$ others supported conservative therapy first with sitz baths with or without addition of antibiotics. ${ }^{713)}$ Further controversy includes necessity and ideal timing for surgery in case of failed conservative treatment. ${ }^{6,7)}$ Further studies will be required to create standardized guidelines for treatment.

The aim of the study was to approve clinic intern management of perianal abscess and fistula in ano in children, evaluate clinical characteristics and identify parameters that predict especially

\footnotetext{
Corresponding author: Lars Boenicke, MD, PhD. Department of General and Visceral Surgery, Helios University Hospital Wuppertal, University Witten-Herdecke, Heusnerstraße 40, D-42283 Wuppertal, Germany 


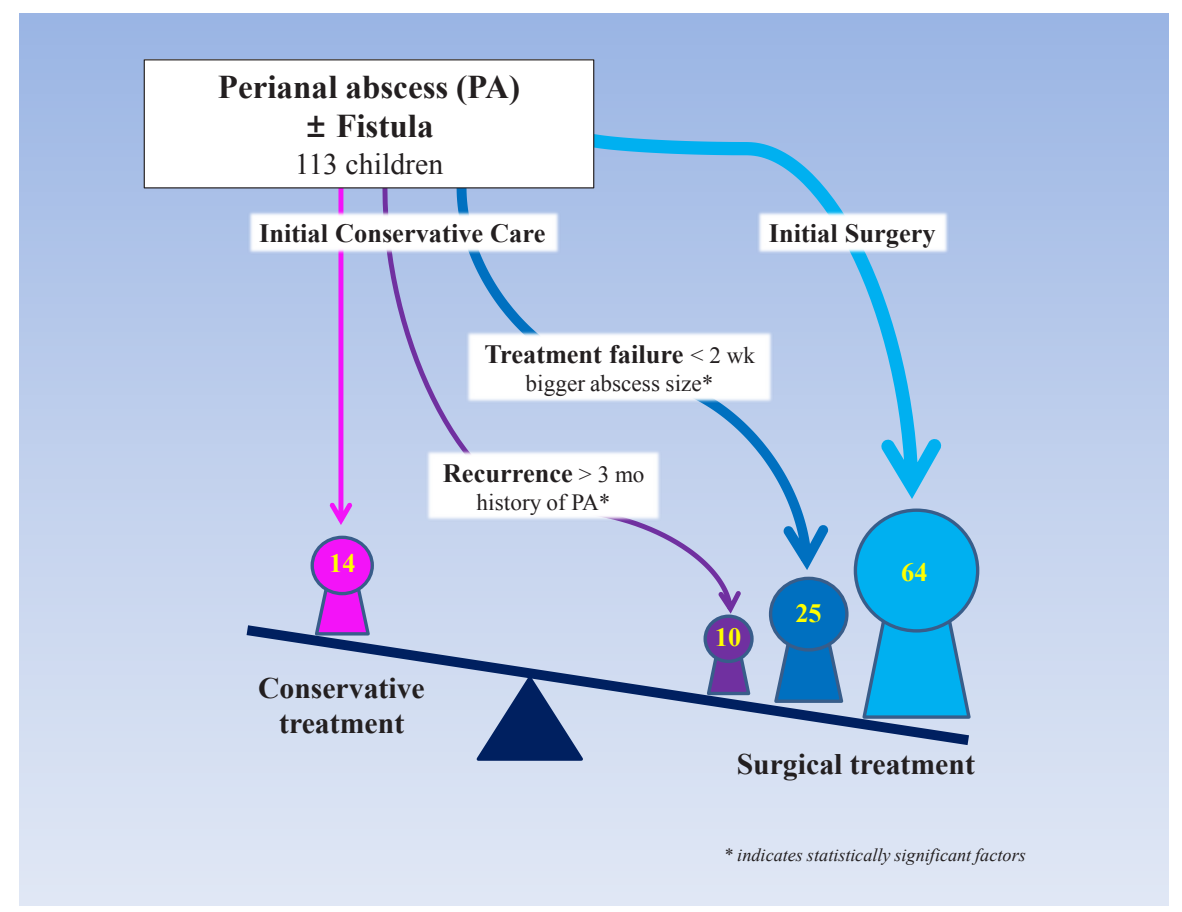

Graphical abstract. Clinical course and outcome of children with perianal abscesses after conservative and surgical treatment.

failure of conservative therapy.

\section{Methods}

\section{Study population}

We conducted a retrospective analysis of children below 14 years treated for perianal abscess in our institution from January 2001 to December 2016. Data was derived from patient records of the medical data base of the clinic, which also included data of the outpatient department. Demographic information of the patients, number and localization of the lesions, clinical, microbiological and laboratory data, usage and duration of antibiotics, abscess recurrences and postoperative follow-up need of surgical attempt were analyzed. Patients with inflammatory bowel diseases and anorectal malformations were excluded from the study.

\section{Treatment algorithm}

Perianal abscess was defined as an infective process in the perianal area with a definite presence of a liquid formation seen at clinical and ultrasound examination. Patients with erythema and induration alone with no definite liquid formation were not included in the study.

In cases of completely encapsulated abscess seen at ultrasound examination, patients were subsequently referred for surgery. The standard procedure for surgery for perianal abscess was incision and drainage in general anesthesia. Probing for fistula was generally only performed if the abscess was recurrent or a discharge of pus was identified from the anal verge during surgery. In case of anal fistula detection, a fistulotomy was performed.

All other cases were treated conservatively first by local care (hygiene and sitz baths) and intravenous antibiotics. Within the first 2 weeks, a daily reassessment was performed in consensus of pediatrics with surgeons to decide whether conservative treatment was continued or patients were referred for surgery. The main reasons for termination of conservative treatment were persisting or increasing complaints like pain or fever. In cases with phlegmon findings at abscess drainage, antibiotics were given postoperatively.

\section{Follow-up}

Follow-up information concerning abscess healing or recurrence at least 3 months after discharge from the hospital was collected from patient records. In patients who had no contact with the hospital after discharge the family physician and/or parents were contacted by telephone interview.

\section{Statistical analysis}

Statistical analysis was performed with IBM SPSS ver. 25.0 (IBM Corp., Armonk, NY, USA). The primary outcome measure was failure of conservative therapy and abscess recurrence and/or fistula formation, respectively, at follow-up. Differences between the success and failure group were evaluated using $\mathrm{McNemar}$ test for categorical variables and the $t$-test for continuous variables.

Multivariate logistic regression yielding odds ratios (ORs) was used to assess significance of preoperative variables for success or failure. All significant risk factors found at univariate analysis were considered for inclusion and entered via stepwise regression ( $P<0.05$ for entry, and $P>0.10$ for exit).

The study protocol was approved by the local ethics com. mittee (AZ06/2018). 


\section{Results}

\section{Study cohort}

From January 2001 to December 2016, 119 patients with perianal abscess were admitted in our institution. Complete records were available for 113 consecutive patients, they were included in the study.

Fig. 1 shows patient history recorded at first admission in our hospital and follow-up of the study cohort. Sixty-four patients had fully encapsulated abscess at admission and were subsequently referred for surgery (primary surgery group). Fortynine patients underwent conservative treatment first and received systemic antibiotics (primary conservative group).

Demographical and clinical characteristics of the 2 groups are shown in Table 1 . There were no significant differences between the primary surgery and the primary conservative group in terms of age, sex and in the majority of anamnestic and clinical parameters. However, in patients of the primary surgery group, the parameters history of perianal abscess $(P=0.037)$ and abscess size $(P=0.001)$ were significantly higher.

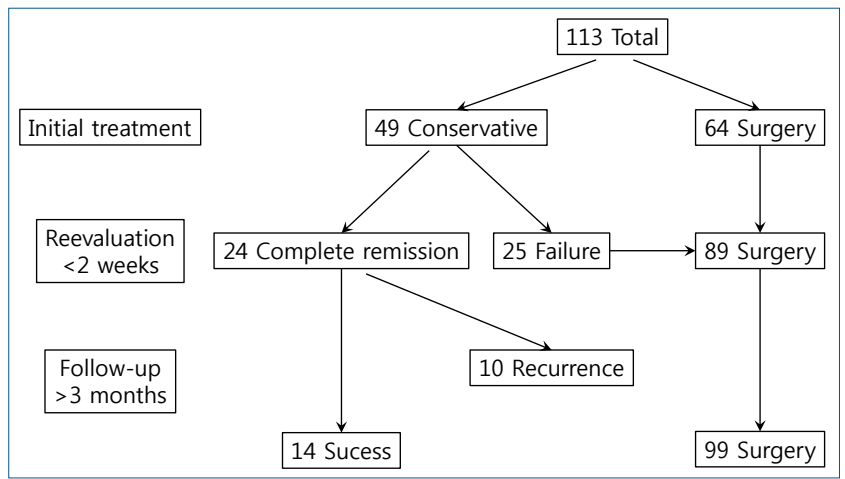

Fig. 1. Follow-up of children with perianal abscesses.

\section{Clinical course and follow-up primary conservative group}

Follow-up is shown in Fig. 1. In the primary conservative group, a complete remission was seen in 24 patients after median 5.2 days (range, 3-12 days). In 25 patients, conservative treatment was considered as inefficient and stopped after median 7.03 days (range, 2-16 days), they were referred for surgery. Thus, initial conservative treatment within the first 2 weeks was effective in 24 of 49 patients (48\%).

However, at mid- and long-term follow-up of median 34 months (range, 3-145 months), 10 of the 24 patients with primary remission under conservative treatment were admitted with recurrent abscess and/or anal fistula, they were referred for surgery. Recurrent disease occurred after median 3 months (range, 1-9 months). Thus, of the entire study cohort of 113 patients, 99 patients underwent surgery and only 14 were successfully treated by conservative treatment alone.

\section{Intraoperative findings and follow-up after surgery}

Finally, 99 patients underwent surgery. In 16 of the patients (16\%), a fistula was detected intraoperatively and a fistulotomy was performed. Thirty-six patients received postoperatively antibiotics for $7.06 \pm 3.03$ days. Follow-up after surgery was 21 months (range, 2-113 months). Recurrent disease requiring surgery occurred in 27 patients (27\%), in 11 of $49(22 \%)$ of the primary conservative group and 16 of $64(25 \%)$ of the primary surgery group. This was not significant $(P=0.75)$. The point of time recurrent disease occurred was median 5 months (range, 1-18 months). In the primary conservative group, it was 4 months (range, 1-18 months) and in the primary surgery group, it was 5 months (range, 2-16 months). This was not significant $(P=0.51)$.

Table 1. Demographic and clinical characteristics of patients with perianal abscesses

\begin{tabular}{|c|c|c|c|c|}
\hline Characteristic & All & Surgery $y^{a)}$ & Conservative $^{\text {b) }}$ & $P$ value \\
\hline Patient & 113 & 64 & 49 & - \\
\hline Age (yr) & $4.64 \pm 4.90$ & $5.09 \pm 5.10$ & $3.70 \pm 4.50$ & 0.051 \\
\hline Height (cm) & $107.60 \pm 4.00$ & $115.07 \pm 42.00$ & $99.53 \pm 41.00$ & 0.091 \\
\hline Weight (kg) & $21.40 \pm 20$ & $24.43 \pm 23$ & $18.15 \pm 18.00$ & 0.131 \\
\hline Male sex & $99(87)$ & $54(84)$ & $45(91)$ & 0.472 \\
\hline Migrant background & $45(39)$ & $27(42)$ & $18(36)$ & 0.432 \\
\hline History of perianal abscess & $21(18)$ & $16(25)$ & $5(10)$ & 0.037 \\
\hline Localization dorsal & $34(30)$ & $23(35)$ & $11(22)$ & 0.098 \\
\hline Localization ventral & $34(30)$ & $22(34)$ & $12(24)$ & 0.216 \\
\hline Localization lateral & $39(34)$ & $18(28)$ & $21(42)$ & 0.251 \\
\hline Localization multifocal & $7(6)$ & $5(7)$ & $2(4)$ & 0.396 \\
\hline Enhanced inflammatory values (CRP and WBC) & $29(25)$ & $18(28)$ & $11(22)$ & 0.438 \\
\hline Temperature $\left(^{\circ}\right)$ & $37.2 \pm 0.6$ & $37.2 \pm 0.6$ & $37.1 \pm 0.6$ & 0.815 \\
\hline Size (mm) & $14.3 \pm 9.6$ & $17.8 \pm 11.1$ & $10.0 \pm 5.1$ & 0.001 \\
\hline
\end{tabular}

Values are presented as mean \pm standard deviation or number (\%).

CRP, C-reactive protein; WBC, white blood count.

Two-sided $t$ test and Fisher exact test were used.

Boldface indicates a statistically significant difference with $P<0.05$.

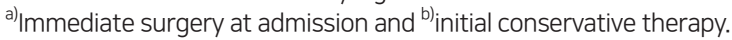


Table 2. Preoperative parameters of patients who initially received conservative treatment for perianal abscesses

\begin{tabular}{|c|c|c|c|c|c|c|}
\hline \multirow{2}{*}{ Parameter } & \multicolumn{3}{|c|}{ Conservative treatment } & \multicolumn{2}{|c|}{$P$ value } & \multirow{2}{*}{ Odds ratio } \\
\hline & All & Success & Failure & Univariate $^{\mathrm{a})}$ & Multivariate & \\
\hline Patient & 49 & 14 & 35 & - & - & - \\
\hline Age (yr) & $3.70 \pm 4.5$ & $3.92 \pm 5.9$ & $3.62 \pm 3.9$ & 0.839 & NS & - \\
\hline Height (cm) & $99.53 \pm 41$ & $100.6 \pm 40$ & $99.17 \pm 41$ & 0.912 & NS & - \\
\hline Weight (kg) & $18.15 \pm 8$ & $18.60 \pm 18$ & $17.97 \pm 18$ & 0.911 & NS & - \\
\hline Male sex (\%) & $45(91)$ & $14(100)$ & $31(88)$ & 0.194 & NS & - \\
\hline Migrant background & $18(36)$ & $5(35)$ & $13(37)$ & 0.927 & NS & - \\
\hline History of perianal abscess & $5(10)$ & $0(0)$ & $5(14)$ & 0.141 & NS & - \\
\hline Localization dorsal & $11(22)$ & $4(28)$ & $7(20)$ & 0.526 & NS & - \\
\hline Localization ventral & $12(24)$ & $2(14)$ & $10(28)$ & 0.303 & NS & - \\
\hline Localization lateral & $24(48)$ & $4(28)$ & $20(57)$ & 0.073 & NS & - \\
\hline Localization multifocal & $2(4)$ & $0(0)$ & $2(5)$ & 0.372 & NS & - \\
\hline Enhanced inflammatory values (CRP and WBC) & $11(22)$ & $0(0)$ & $11(31)$ & 0.017 & 0.124 & 0.808 \\
\hline Temperature $\left(^{\circ}\right)$ & $37.1 \pm 0.6$ & $37.0 \pm 0.2$ & $37.2 \pm 0.7$ & 0.200 & NS & - \\
\hline Size (mm) & $10.0 \pm 5.1$ & $6.35 \pm 4.2$ & $11.48 \pm 4.3$ & 0.001 & 0.023 & 3.37 \\
\hline
\end{tabular}

Values are presented as mean \pm standard deviation or number (\%).

NS, not significant; CRP, C-reactive protein; WBC, white blood count.

Two-sided $t$ test and Fisher exact test were used. Multivariate log regression analysis.

Boldface indicates a statistically significant difference with $P<0.05$.

${ }^{a}$ Univariate and multivariate analyses of therapy failure.

Table 3. Pre- and intraoperative parameters of patients who underwent surgery for anal abscesses

\begin{tabular}{|c|c|c|c|c|c|c|}
\hline \multirow{2}{*}{ Parameter } & \multicolumn{3}{|c|}{ Patient who underwent surgery } & \multicolumn{2}{|c|}{$P$ value ${ }^{a)}$} & \multirow{2}{*}{ Odds ratic } \\
\hline & All & Success & Failure & Univariate & Multivariate & \\
\hline Patient & 99 & 72 & 27 & - & - & - \\
\hline Mean age (yr) & $4.64 \pm 4.78$ & $5.24 \pm 4.82$ & $3.06 \pm 4.37$ & 0.043 & 0.160 & 1.38 \\
\hline Male sex & $86(87)$ & $61(85)$ & $25(93)$ & 0.505 & NS & - \\
\hline Migrant background (\%) & $43(43)$ & $35(49)$ & $8(30)$ & 0.113 & NS & - \\
\hline History of perianal abscess & $21(21)$ & $8(11)$ & $13(48)$ & 0.000 & 0.043 & 4.13 \\
\hline Localization dorsal & $31(31)$ & $20(28)$ & $11(41)$ & 0.232 & NS & - \\
\hline Localization ventral & $32(32)$ & $23(32)$ & $9(33)$ & 1.00 & NS & - \\
\hline Localization lateral & $36(36)$ & $26(36)$ & $10(37)$ & 1.00 & NS & - \\
\hline Localization multifocal & $7(7)$ & $2(3)$ & $5(19)$ & 0.015 & 0.475 & 3.77 \\
\hline Temperature $\left(^{\circ}\right)$ & $37.23 \pm 0.75$ & $37.28 \pm 0.65$ & $37.12 \pm 0.69$ & 0.299 & NS & - \\
\hline Size (mm) & $15.89 \pm 9.74$ & $16.50 \pm 10.48$ & $14.26 \pm 7.38$ & 0.238 & NS & - \\
\hline Preoperative antibiotics & $35(35)$ & $25(34)$ & $10(37)$ & 0.617 & NS & - \\
\hline
\end{tabular}

Values are presented as mean \pm standard deviation or number (\%).

NS, not significant.

Two-sided $t$ test, Fisher exact test, and logistic regression analyses were conducted.

Boldface indicates a statistically significant difference with $P<0.05$.

a) Univariate and multiple analyses of therapeutic failure.

\section{Risk factors for failure of conservative therapy}

Univariate analysis for risk factors for failure of conservative treatment is shown in Table 2. Enhanced inflammatory values $(P=0.017)$ and size of the abscess $(P=0.001)$ were significantly higher in patients with failure of conservative treatment. There were no significant differences in age, sex, or clinical parameters. During multivariate log regression analysis, only abscess size $(\mathrm{OR}, 3.37 ; P=0.023)$ was an independent parameter for therapy failure.

\section{Risk factors for recurrent disease after surgery}

Univariate analysis for risk factors for recurrent disease after surgery is shown in Table 3. Of the parameters evaluated at first admission in our hospital, there were significant differences in age, history of perianal abscess and multilocal localization. During multivariate $\log$ regression analysis, only history of perianal abscess (OR, $4.13 ; P=0.043)$ was an independent parameter for therapy failure.

\section{Subgroup analysis infants vs. older children primary conser- vative group}

Table 4 shows subgroup analysis of infants (age $<2$ years) vs. children older than 2 years of the primary conservative group. Of the parameters that were not associated with age, there were 
Table 4. Demographic and clinical characteristics and outcome of patients with perianal abscesses who initially received conservative treatment for perianal abscesses

\begin{tabular}{|c|c|c|c|c|}
\hline Characteristic & All & Infant (<2 yr) & Age (2 $14 \mathrm{yr})$ & $P$ value \\
\hline Patient & 49 & 29 & 20 & \\
\hline Successful conservative treatment & 14 & $10(34)$ & $4(20)$ & 0.122 \\
\hline Age $(y r)$ & $3.70 \pm 4.50$ & $0.30 \pm 0.30$ & $8.09 \pm 3.70$ & 0.001 \\
\hline Height (cm) & $99.53 \pm 41.00$ & $63.67 \pm 6.60$ & $141.39 \pm 22.40$ & 0.001 \\
\hline Weight (kg) & $18.15 \pm 8$ & $7.07 \pm 2.00$ & $34.21 \pm 19.40$ & 0.001 \\
\hline Male sex & $45(91)$ & $27(90)$ & $18(93)$ & 0.703 \\
\hline Migrant background & $18(36)$ & $12(41)$ & $6(30)$ & 0.427 \\
\hline History of perianal abscess & $5(10)$ & $3(10)$ & $2(10)$ & 0.998 \\
\hline Localization dorsal & $11(22)$ & $5(17)$ & $6(30)$ & 0.302 \\
\hline Localization ventral & $12(24)$ & $4(13)$ & $8(40)$ & 0.036 \\
\hline Localization lateral & $24(48)$ & $18(62)$ & $6(30)$ & 0.027 \\
\hline Localization multifocal & $2(4)$ & $2(6)$ & 0 & 0.391 \\
\hline Enhanced inflammatory values (CRP and WBC) & $11(22)$ & $4(13)$ & $7(35)$ & 0.083 \\
\hline Temperature $\left(^{\circ}\right)$ & $37.2 \pm 0.6$ & $37.08 \pm 0.50$ & $37.51 \pm 0.80$ & 0.044 \\
\hline Size $(\mathrm{mm})$ & $10.0 \pm 5.1$ & $8.28 \pm 5.60$ & $12.68 \pm 4.70$ & 0.006 \\
\hline Recurrent disease after subsequent surgery & $11(22)$ & $8 / 19(42)$ & $3 / 16(18)$ & 0.119 \\
\hline
\end{tabular}

Values are presented as mean \pm standard deviation or number (\%).

CRP, C-reactive protein; WBC, white blood count.

Two-sided $t$ test and Fisher exact test were used.

Boldface indicates a statistically significant difference with $P<0.05$.

significant differences in abscess localization, temperature and abscess size. Permanent success of conservative therapy was achieved in 10 of 29 infants (34\%) and 4 of 20 children (20\%) older than 2 years. However, this was not significant $(P=0.122)$.

\section{Discussion}

Although perianal abscess and fistula are common in children, the management remains controversial. The clinical course and outcome of the pediatric disease differs from the adult population, and an alternative etiology especially in infants has been discussed. ${ }^{6,714)}$ While some authors primarily favor surgery, ${ }^{10-12)}$ others supported conservative therapy first with sitz baths with or without addition of antibiotics. ${ }^{7,13,15,16)}$

In order to find the appropriate management, it would be important to identify parameters that predict success or failure especially of conservative treatment. Our clinic traditionally favors a conservative therapeutic attempt in suitable patients, and surgical treatment is rather recommended in advanced disease. However, in this study, more than half of the patients had fully encapsulated abscess at admission and underwent subsequent surgery.

In the other half of the study cohort, conservative therapy with intravenous antibiotics was initiated.

Although the 2 groups were not randomized, important demographical parameters including age, sex, and clinical findings were similar. The only differences were history of recurrent abscess and abscess size, which were significantly higher in the primary surgery group. Generally spoken, these parameters indicate a more advanced or chronical disease. One can postulate, that patients of the primary conservative group may have an earlier stage of disease with a mixture of both interstitial and liquid inflammation, which may easier respond to antibiotics.

However, even in this selected cohort, initial conservative treatment was only successful in $50 \%$ of the cases, the other half of the patients underwent surgery in the first 2 weeks because of persisting abscess and/or complaints. And even in these initially healed patients, recurrent disease occurred after months in 10 out of 24 cases, showing that a permanent healing after conservative treatment was achieved in only a minority of patients. Therefore, the usefulness of conservative treatment has to be scrutinized.

On the other hand side, later recurrence after surgery was in both the conservative and the primary surgery group similar, showing no disadvantage in the long run when trying a conservative treatment first for some days.

To avoid useless conservative therapeutic attempts, it would be helpful to identify parameters that predict failure of conservative treatment. In a multivariate analysis, we found abscess size as an independent parameter that predicts failure of conservative treatment. Thus, patients with larger perianal abscess should be referred early for surgery.

The question is why results and recommendations for adequate therapy are so heterogeneous in the literature. One reason may be that some studies include only infants, others all children younger than 14 years and that conservative treatment may be more efficient in this special subgroup. However, in the present study, there was only a tendency of higher efficiency of conservative treatment in infants but this was not significant, and age was no predictive parameter for permanent success or failure in the study cohort. Serour and Gorenstein ${ }^{6}$ also found also no significant differences in recurrences when comparing children older than 2 years with infants. 
Chang et al. ${ }^{17)}$ found excellent results after conservative treatment in infants, however, but the mean duration of treatment was almost half of a year, which may be considered to be too long in a potentially painful disease.

Taken together, conservative treatment of perianal abscess with or without anal fistula in children was only in a minority of patients permanently successful in this study. However, recurrence after surgery was not higher in patients who underwent conservative treatment first. In a multivariate analysis, abscess size was a negative predictive factor for therapy failure and might be utilized for identifying appropriate patients for successful conservative treatment.

The limitation of the study might be the retrospective study design and that the groups are not randomized. Cases with early stage disease without abscess formation were excluded from the study and may respond better to conservative therapy.

\section{Conflicts of interest}

No potential conflict of interest relevant to this article was reported.

\section{Acknowledgments}

We would like to thank Dr. Johanna Lass-Hennemnan (University Homburg, Germany) for excellent statistical analysis.

See the commentary "Management of perianal abscess and fistula-in-ano in infants and children" via https://oi.org/ 10.3345/cep.2020.00150.

\section{References}

1. Emile SH, Elfeki H, Abdelnaby M. A systematic review of the mana- gement of anal fistula in infants. Tech Coloproctol 2016;20:735-44.

2. Ommer A, Herold A, Berg E, Fürst A, Post S, Ruppert R, et al. German S3 guidelines: anal abscess and fistula (second revised version). Langenbecks Arch Surg 2017;402:191-201.

3. Nelson R. Anorectal abscess fistula: what do we know? Surg Clin North Am 2002;82:1139-51.

4. Eisenhammer S. The anorectal fistulous abscess and fistula. Dis Colon Rectum 1966;9:91-106.

5. Boenicke L, Karsten E, Zirngibl H, Ambe P. Advancement flap for treat ment of complex cryptoglandular anal fistula: prediction of therapy success or failure using anamnestic and clinical parameters. World J Surg 2017;41:2395-400.

6. Serour F, Gorenstein A. Characteristics of perianal abscess and fistula-inano in healthy children. World J Surg 2006;30:467-72.

7. Poenaru D, Yazbeck S. Anal fistula in infants: etiology, features, management. J Pediatr Surg 1993;28:1194-5.

8. Piazza DJ, Radhakrishnan J. Perianal abscess and fistula-in-ano in children. Dis Colon Rectum 1990;33:1014-6.

9. Afşarlar CE, Karaman A, Tanir G, Karaman I, Yilmaz E, Erdoğan D, et al. Perianal abscess and fistula-in-ano in children: clinical characteristic, management and outcome. Pediatr Surg Int 2011;27:1063-8.

10. Festen $C$, van Harten $H$. Perianal abscess and fistula-in-ano in infants. J Pediatr Surg 1998;33:711-3.

11. Murthi GV, Okoye BO, Spicer RD, Cusick EL, Noblett HR. Perianal abscess in childhood. Pediatr Surg Int 2002;18:689-91.

12. Novotny NM, Mann MJ, Rescorla FJ. Fistula in ano in infants: who recurs? Pediatr Surg Int 2008;24:1197-9.

13. Christison-Lagay ER, Hall JF, Wales PW, Bailey K, Terluk A, Goldstein $\mathrm{AM}$, et al. Nonoperative management of perianal abscess in infants is associated with decreased risk for fistula formation. Pediatrics 2007; 120:e548-52.

14. Meyer T, Weininger M, Höcht B. Perianal abscess and anal fistula in infancy and childhood. A congenital etiology? Chirurg 2006;77:102732.

15. Rosen NG, Gibbs DL, Soffer SZ, Hong A, Sher M, Peña A. The nonoperative management of fistula-in-ano. J Pediatr Surg 2000;35:938-9.

16. Oh JT, Han A, Han SJ, Choi SH, Hwang EH. Fistula-in-ano in infants: is nonoperative management effective? J Pediatr Surg 2001;36:1367-9.

17. Chang HK, Ryu JG, Oh JT. Clinical characteristics and treatment of perianal abscess and fistula-in-ano in infants. J Pediatr Surg 2010;45: 1832-6. 